\title{
Cloning and Expression of Cadherin Fragment from Helicoverpa armigera
}

\author{
K. Momena ${ }^{1}$, P. A. Kumar ${ }^{2}$, H. Mehraj ${ }^{3}$ and A. F. M Jamal Uddin ${ }^{3 *}$ \\ Advanced Seed Research and Biotech Center, ACI Limited, Dhaka, Bangladesh ${ }^{1}$ \\ National Research Center on Plant Biotechnology, IARI, New Delhi, India ${ }^{2}$ \\ Dept. of Horticulture, Sher-e-Bangla Agricultural University, Dhaka, Bangladesh ${ }^{3}$ \\ Corresponding author": jamal4@yahoo.com
}

\begin{abstract}
Helicoverpa armigera is an important pest of agricultural crops which causes severe damage to the fruits and pods of crop plants, larval stage which causes this kind of damage. Helicoverpa armigera cadherin fragment contains its toxin binding region which enhance crylAc activity against Helicoverpa armigera larvae because this kind of interaction between HaCadl receptors and crylAc induces toxin oligomerization. The aim of current investigation was to clone and expression the cadherin fragment. Cadherin gene was isolated from mid gut portion of Helicoverpa armigera and cloned into protein expression vector pMALc $2 x$ for expression study. The recombinant clone was transformed into protein expression host and protein was isolated and analysed the expected $k D a$ by SDS- PAGE and Western blotting. The protein band was $70 \mathrm{kDa}$ that confirmed $26.9 \mathrm{kDa}$ cadherin protein was fused with $42.5 \mathrm{kDa}$ Maltose binding protein. Present study successfully cloned and expressed cadherin fragment HaCadl and CrylAc protein in Helicoverpa armigera. Our result provided a tactic for cadherin gene cloning and transformation into crylAc transgenic plants that can significantly enhance the insecticidal activity of CrylAc against Helicoverpa armigera.
\end{abstract}

Key words: Helicoverpa armigera, CrylAc insecticidal protein and cadherin

\section{Introduction}

Pest management has always been an important challenge to agricultural community. Crop yields are substantially reduced by insect pests that attack them. Bt crystal proteins are the first insecticidal protein for introduction into plants because of their environmental safety. A variety of genes encoding for different classes of insecticidal proteins such as protease inhibitors, lectins, amylase inhibitors, scorpion-venom toxins, cytokinin biosynthesis enzymes, cholesterol oxidase, chitinase and $\delta$ endotoxins of Bacillus thuringiensis are being tested for insect control. Among these, the insecticidal crystal proteins of $B t$ assume major significance due to their potency, insect specificity and lack of activity in mammals and other organisms (Kumar et al., 1996). Bacillus thuringiensis is a ubiquitous gram-positive, spore forming bacterium. It produces parasporal insecticidal crystal proteins (ICPs) also known as Cry proteins which exhibit toxicity to certain insect species. These insecticidal crystal proteins (ICPs) display insect specific toxicity and are harmless to humans and non-target animals. Consequently, they have been widely used in biopesticides and transgenic plants to control agricultural pests (Schnepf et al., 1998; Pigott and Ellar, 2007). Bacillus thuringiensis is already a useful alternative or supplement to synthetic chemical pesticide. Cadherin belongs to one of the families of animal glycoproteins responsible for calcium-dependent cell-cell adhesion, Cadherin (named for calcium dependent adhesion). The interaction between Bacillus thuringiensis insecticidal crystal protein Cry1 A and Cadherin receptors in lepidopteran insects induces toxin oligomerization, which is essential for membrane insertion and mediates Cry1A toxicity. They are dependent on 
calcium $\left(\mathrm{Ca}^{2+}\right)$ ions to function, hence their name. Recent literature showed that the Cadherin-like protein in midgut of several insects served as the receptor of $B t$ toxin Cry1A and variation of Cadherin-like protein is related to insect's resistance to crylA. cDNA sequence of a cadherin gene in Helicoverpa armigera ( $\mathrm{HaCad}$ ) and the crylAc toxin binding region (TBR) to residues 1217-1461 (Wang et al., 2005). Now a days cloning of the cadherin fragment is a novel strategy to enhance insecticidal activity or to overcome the resistance of insects. As Helicoverpa armigera is a major crop pest, cloning of cadherin gene fragment into the protein expression vector can help to manage this pest. The view of the present study was to clone the receptor region of $\mathrm{HaCadl}$ into E .coli expression vector pMALc2X and analyze the protein by SDS- PAGE and Western blotting.

\section{Material and Methods}

An experiment was conducted in National Research Center on Plant Biotechnology, Indian Agricultural Research Institute, New Delhi, India with a view for designing of primers for amplification of HaCadl from Helicoverpa armigera: Based on published HaCadl (Genebank no.DQ973295 - Peng et al., 2009) gene sequences, a set of specific primers were used to amplify the receptor binding portion of cadherin from the cDNA sample of Helicoverpa armigera midgut portion.

Total RNA isolation from $\boldsymbol{H}$. armigera mid gut: Total RNA was isolated from the mid gut portion of the $4^{\text {th }}$ instar larvae of the Helicoverpa armigera. RNA isolation was done by using TRizol reagent (Invitrogen). Quality and quantity of the total RNA was checked by gel electrophoresis and Nanodrop 1000 spectrophotometer respectively (Figure 01).

cDNA synthesis from the total RNA: Single stranded cDNA was prepared by using AffinityScript QPCR cDNA Synthesis Kit. (Stratagene) (Figure 02). The following reaction components were added in microcentrifuge tube in order:

\begin{tabular}{lc}
\hline RNase-free $\mathrm{H}_{2} \mathrm{O}$ & $5.0 \mu \mathrm{l}$ \\
Total RNA $(1 \mu \mathrm{g})$ & $1.0 \mu \mathrm{l}$ \\
First strand master mix $(2 \times)$ & $10.0 \mu \mathrm{l}$ \\
Oligo (dT) primer $(0.1 \mu \mathrm{g} / \mu \mathrm{l})$ & $3.0 \mu \mathrm{l}$ \\
AffinityScript RT/RNase Block enzyme mixture & $1.0 \mu \mathrm{l}$ \\
\hline
\end{tabular}

The above reaction was kept in thermal cycler and followed the condition according to the manufacturer's instruction. After cDNA synthesis the quality and quantity of the cDNA was checked on $1.2 \%$ agarose gel and Nanodrop respectively.

Amplification of the cadherin gene fragment: Gradient PCR was performed to find out the annealing temperature for amplification of cadherin receptor region at $50-60^{\circ} \mathrm{C}$ by using Taq DNA polymerase enzyme (Bangalore Genei). Pfu DNA polymerase was used to amplify the final product and avoid the mismatch of the nucleotide sequences. Amplification was performed in an eppendorf thermal cycler (Eppendorf AG, Germany) (Figure 03).

Analysis and Purification of PCR products: After amplification of cDNA, it was checked in 1\% agarose gel electrophoresis and purified the cDNA using QIAquick PCR purification kit (QIAGEN, Germany).

Cloning of cadherin gene (HaCad1) fragment into pMALc2x expression vector: For the expression of cadherin gene, 732 bp of PCR purified cadherin gene was restricted with BamHI and SacI site and cloned into correspondent site of pET29 (a+) vector. The cadherin fragment was further cloned into pMALc2X expression vector by BamHI - HindIII restriction sites for improving the 
protein expression. Routine lab protocols were followed for ligation, transformation and screening of transformed colonies (Figure 04).

Overexpression of cadherin protein: The clone was transformed into the TB1 host cells and the transformants were used for screening. Protein was isolated according to manufacture instruction (New England BioLabs) from single colony and induced by $1 \mathrm{mM}$ IPTG. The overexpressed protein was checked by SDS-PAGE and Western blotting.

Screening for overexpression colony by SDS-PAGE analysis and Western Blotting: Isolated protein was checked on 12\% SDS-PAGE (BIO-RAD Mini-Protein-3 Electrophoresis system). Because of the small fragment of the cadherin gene the expected band size was not clearly seen under the SDS-PAGE. For further confirmation, western blotting was carried out. SDS-PAGE separated proteins were transferred to nitro cellular membrane using mini trans-blot electrophoretic transfer cell apparatus (BIO-RAD, USA). After adding primary and secondary antibody to the membrane, it was further washed with PBST for three times and air dried on what man paper and immediately photograph was taken (Figure 05a and 05b).

\section{Results}

\section{Experimental setup and cloning of cadherin gene}

Based on published HaCad1 (Gene Bank no. DQ973295-Peng et al., 2009) gene sequence, set of primers were designed for amplification of receptor binding portion of cadherin fragment. Total RNA was isolated from the larval mid gut (Figure 01) and converted into single stranded cDNA (Figure 02) and used as template for PCR amplification (Figure 03). 732bp PCR amplified cDNA fragment (toxin binding region) was cloned into the protein expression vector. PCR amplified $732 \mathrm{bp}$ gene fragment was restricted with $\mathrm{BamHI}$ and $\mathrm{SaCI}$ and cloned into the corresponding sites of protein expression vector pET29a (+). Cadherin fragment was inserting out from pET29a (+) vector using BamHIHindIII sites and further cloned into pMALc2x expression vector (Figure 04) for improving the expression. The clone was further confirmed by multiple restriction digestion analysis.

\section{Analysis of protein expression by SDS-PAGE and Western Blotting}

PMALc2X-cadherin vector were transformed into TB1 E. coli host to express the protein. Since the gene fragment was extremely small consisting of 732bp and the proteins molecular weight being only $26.9 \mathrm{kDa}$ it which was giving a very faint band on the SDS-PAGE so it was decided that this protein would be cloned into pMALC2X vector which contains a maltose binding fusion protein which is $42.5 \mathrm{kDa}$. After cloning both proteins fuse together and yielded $70 \mathrm{kDa}$ fusion protein was found in SDS PAGE (Figure 05a). For further confirmation western blotting was carried out and a 70kDa fusion protein was obtained which contains $26.9 \mathrm{kDa}$ of cadherin protein along with $42.5 \mathrm{kDa}$ of maltose binding protein (Figure 05b). 


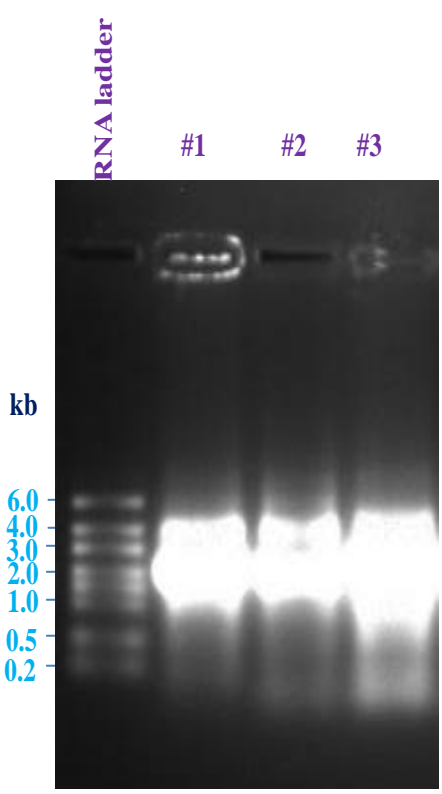

1.2\% Formaldehyde Agarose gel

Figure 01. Analysis of total RNA from mid gut portion of $H$. armigera

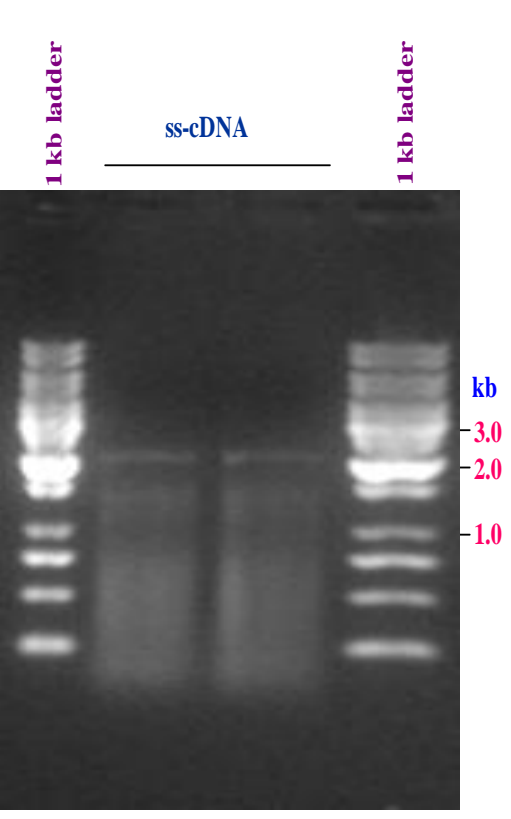

1.2\% Agarose gel

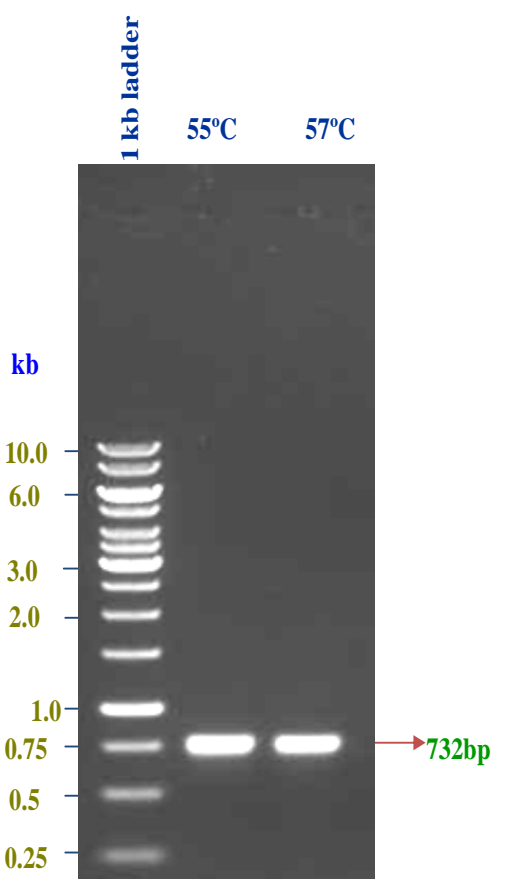
Figure 02. Synthesis of cDNA Figure 03. PCR amplification of
from midgut total RNA of $\mathrm{H}$. cadherin gene fragment using armigera cDNA sample

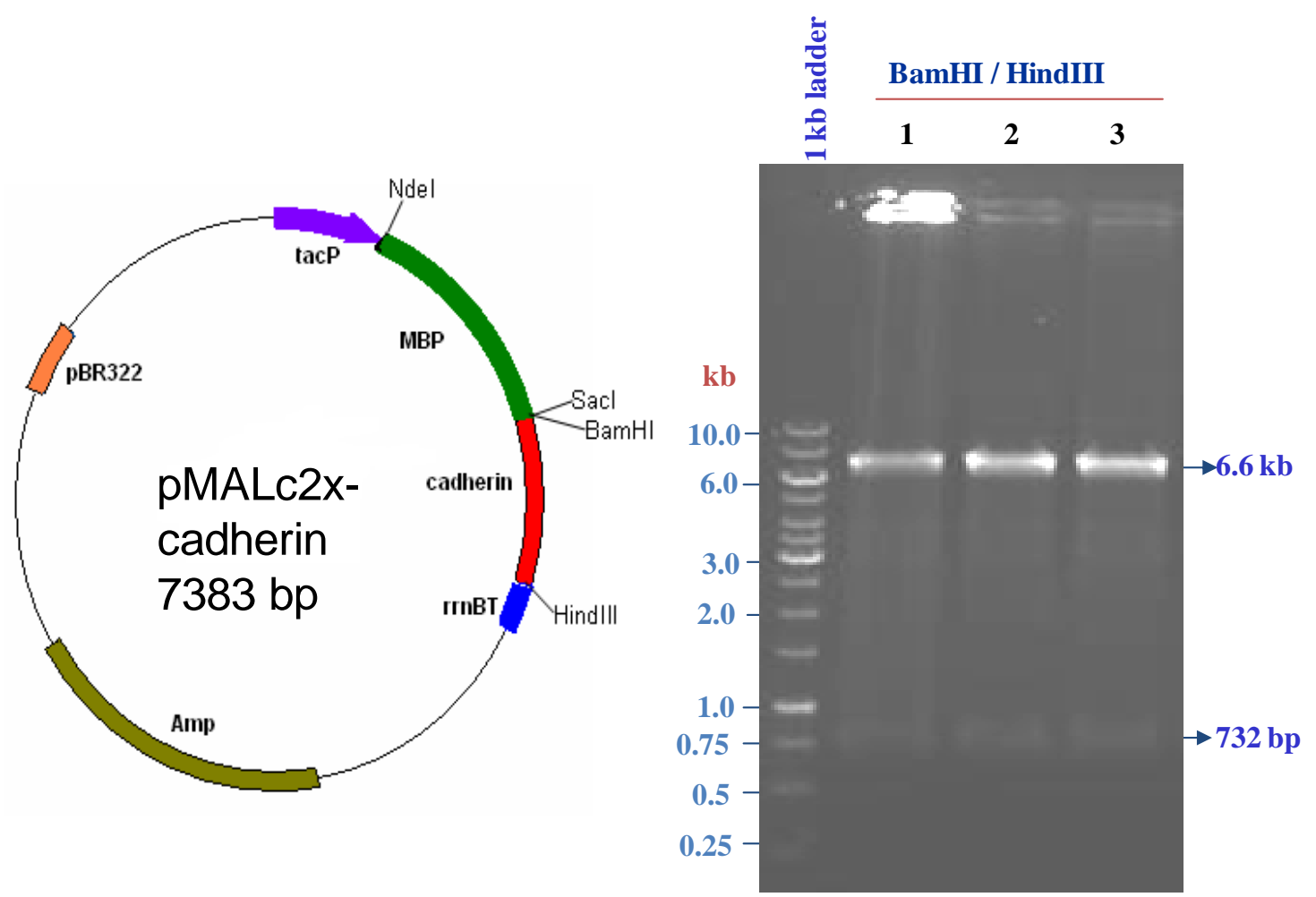

Figure 04. Construction and restriction digestion analysis of pMALc2x protein expression vector carrying cadherin gene 


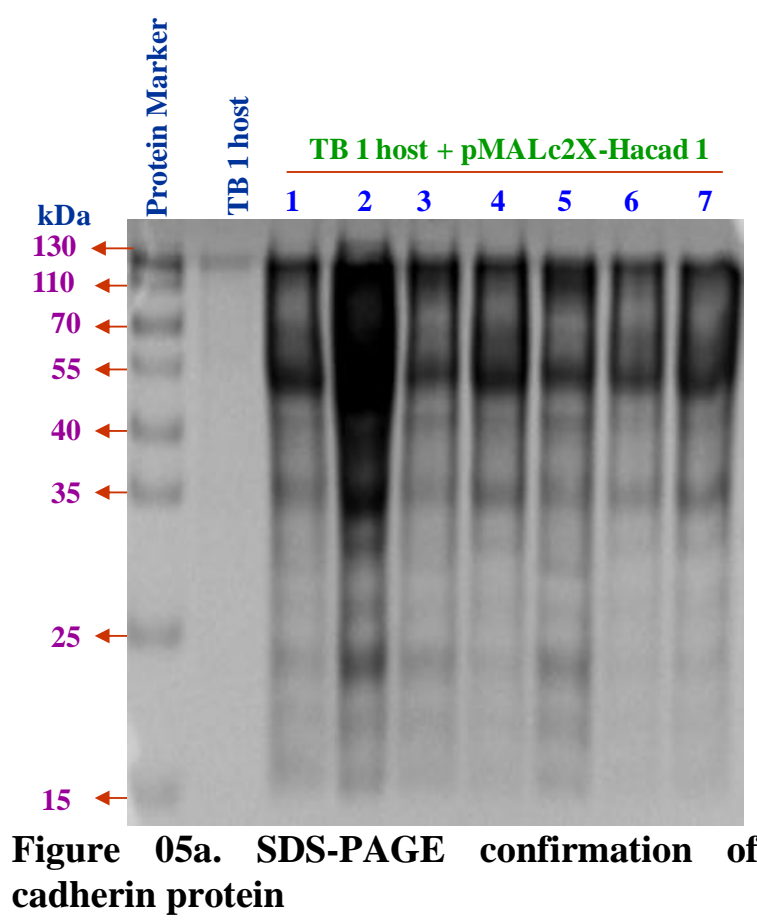

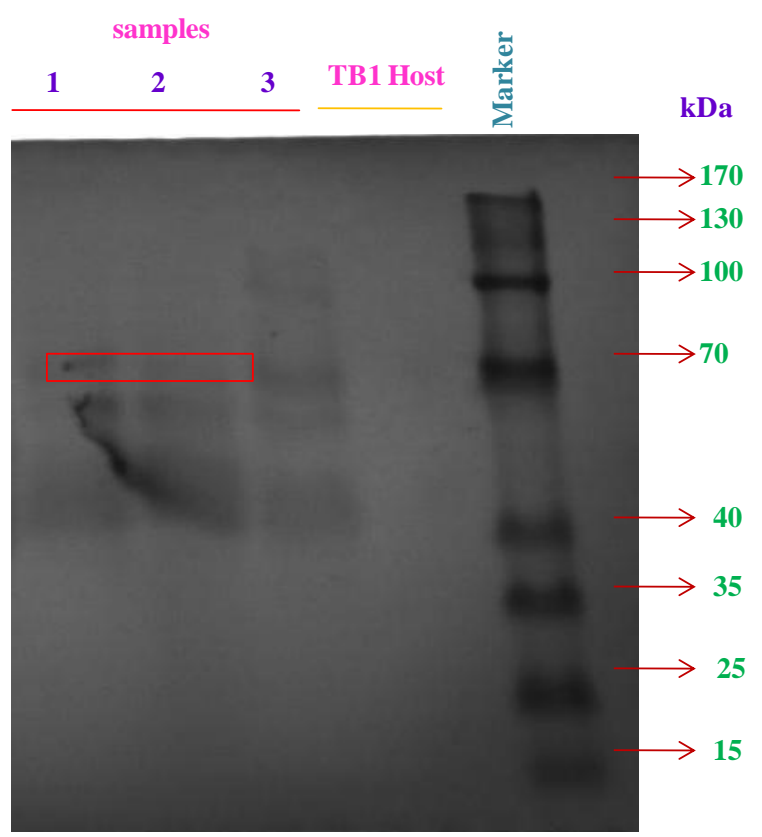

Figure 05b. Western blotting confirmation of cadherin protein

\section{Discussion}

Genetically engineered crops to produce $\mathrm{Bt}$ toxins for insect control have reduced the use of conventional insecticides. Bacillus thuringiensis produces insecticidal crystal protein. Previously, toxin binding region of Helicoverpa armigera cadherin fragment ( $\mathrm{HaCadl}$ ) cloning into binary vector and retransformation into the $c r y l A c$ plants through Agrobacterium mediated plant transformation was done by Momena et al. (2014). The cry1Ac toxin binding sites of HaCad have been mapped to residues 1217 to 1461 by Wang et al. (2005) and found that HaCad1 enhanced the insecticidal activity of Bacillus thuringiensis cry1Ac toxins to Helicoverpa armigera. A critical step in the mode of action of cry proteins is binding of toxins to mid guts membrane receptors (Schnepf et al., 1998; Whalon and Wingerd, 2003). It was proposed that midgut epithelium receptors were involved in insect toxicity by interacting sequentially with the cry1A toxin (Bravo et al., 2004 and 2007; Jurat- Fuentes and Adang, 2006; Gomez et al., 2007). Cadherin belongs to one of the families of animal glycoproteins responsible for calcium-dependent cell-cell adhesion hence, named cadherin. The interaction between Bacillus thuringiensis insecticidal crystal protein Cry1A and cadherin receptors in lepidopteran insects induces toxin oligomerization, which is essential for membrane insertion and mediates Cry $1 \mathrm{~A}$ toxicity. Based on these previous reports the present study has done to demonstrate the amplification of receptor binding portion of cadherin fragment from $H$. armigera, cloning to protein expression vector and over express the protein, analysed by SDS-PAGE and western blotting.

In SDS-PAGE analysis it shown very faint band due to low molecular weight of protein, so that it was further confirmed by western blot analysis. The result shows that $70 \mathrm{kDa}$ protein band on the SDSPAGE and western blot, which indicates $26.9 \mathrm{kDa}$ cadherin proteins, was fused with $42.5 \mathrm{kDa}$ Maltose binding protein. 


\section{Conclusion}

The present study showed that 732 bps cadherin gene fragment was successfully cloned into the protein expression vector pMALc2X. Over expressed protein was analysed and confirmed by SDSPAGE and western blot, it shows $70 \mathrm{kDa}$ protein band which confirmed by western blot, which indicates $26.9 \mathrm{kDa}$ cadherin protein was fused with $42.5 \mathrm{kDa}$ Maltose binding protein. To study the toxin enhancement of the cry1Ac, cadherin gene can be cloned into plant transformation vector and retransformed into cry1Ac transgenic plants.

\section{References}

Bravo, A., Gill, S. S. and Soberon, M. (2007). Mode of action of Bacillus thuringiensis toxins and their potential for insect control. Toxicon. 49: 423-435.

Bravo, A., Gomez, I., Conde, J., Munoz-Garay, C., Sanchez, J., Miranda, R., Zhuang, M., Gill, S. S. and Soberon, M. (2004). Oligomerization triggers binding of a Bacillus thuringiensis Cry1 Ab pore-forming toxin to aminopeptidase $\mathrm{N}$ receptor leading to insertion into membrane microdomains. Biochim. Biophys. Acta. 1667: 38-46.

Gomez, I., Pardo-Lopez, L., Munoz-Garay, C., Fernandez, L. E., Perez, C., Sanchez, J. Soberon, M. and Bravo, A. (2007). Role of receptor interaction in the mode of action of insecticideal Cry and Cyt toxins produced by Bacillus thuringiensis. Peptides. 28: 169-173.

Jurat-Fuentes, J. L., and Adang, M. J. (2006). The Heliothis virescens cadherin protein expressed in Drosophila S2 cells functions as a receptor for Bacillus thuringiensis Cry1A, but not Cry1Fa toxins. Biochemistry, 45(32): 9688-9695.

Kumar, P. A., Sharma, S. R. and Malik, V. S. (1996). Insecticidal proteins of Bacillus thuringiensis. Adv. Appl. Microbio. 42: 1-43.

Momena, K., Kumar, P. A., Mehraj, H. and Jamal Uddin A. F. M. (2014) Cloning of cadherin gene into plant binary vector and retransformation into crylAc transgenic tobacco. Int. J. Sustain. Crop Prod. 9(3): 27-34.

Peng, D. H., Luo, Y., Guo, S. X., Zeng, H., Ju, S. Y., Yu, Z. N. and Sun, M. (2009). Elaboration of an electroporation protocol for large plasmids and wild-type strains of Bacillus thuringiensis. $J$. Appl. Microbiol. 106: 1849-1858.

Pigot, C. R. and Ellar, D. J. (2007). Role of receptors in Bacillus thuringiensis crystal toxin activity. Microbiol. Mol. Biol. Rev. 71: 255-281.

Schnepf, E., Crickmore, N., van Rie, J., Lereclus, D., Baum, J., Feitelson, J., Zeigler, D. R. and Dean, D. H. (1998). Bacillus thuringiensis and its pesticidal proteins. Microbiol. Mol. Biol. Rev. 62: 775-806.

Wang, G. R., Liang, G. M., Wu, K. M. and Guo, Y. Y. (2005). Gene cloning and sequencing of aminopeptidase N3, a putative receptor for Bacillus thuringiensis insecticidal Cry1Ac toxin in Helicoverpa armigera (Lepidoptera: Noctuidae). Eur. J. Entomol. 102: 13-19.

Whalon, M. E. and Wingerd, B. A. (2003). Bt: mode of action and use. Arch. Insect Biochem. Physiol. 54: $200-211$.

\section{Citation for this article (APA Style):}

Momena, K., Kumar, P. A., Mehraj, H. \& Jamal Uddin, A. F. M. (2015). Cloning and expression of Cadherin fragment from Helicoverpa armigera. Journal of Bioscience and Agriculture Research 03(01), 59-64.

Retrieved March 10, 2015 from http://www.journalbinet.com/jbar-volume-03-issue-01.html 